Article

\title{
Quantum Estimates of Ostrowski Inequalities for Generalized $\phi$-Convex Functions
}

\author{
Miguel J. Vivas-Cortez ${ }^{1, *,+}$ (1), Artion Kashuri ${ }^{2,+}$ (D), Rozana Liko ${ }^{2,+}$ (i) and \\ Jorge E. Hernández Hernández ${ }^{3,+}$ \\ 1 Escuela de Ciencias Físicas y Matemáticas , Facultad de Ciencias Exactas y Naturales, Pontificia Universidad \\ Católica del Ecuador, Av. 12 de Octubre 1076. Apartado, Quito 17-01-2184, Ecuador \\ 2 Department of Mathematics, Faculty of Technical Science, University Ismail Qemali, L. Pavaresia, \\ Vlora 1001, Albania; artionkashuri@gmail.com (A.K.); rozanaliko86@gmail.com (R.L.) \\ 3 Departamento de Técnicas Cuantitativas, Decanato de Ciencias Económicas y Empresariales, \\ Universidad Centroccidental Lisandro Alvarado, Av. 20. esq. Av. Moran, Edf. Los Militares, Piso 2, Ofc.2, \\ Barquisimeto 3001, Venezuela; jorgehernandez@ucla.edu.ve \\ * Correspondence: mjvivas@puce.edu.ec \\ + These authors contributed equally to this work.
}

Received: 4 November 2019; Accepted: 4 December 2019; Published: 12 December 2019

\begin{abstract}
In this paper, the study is focused on the quantum estimates of Ostrowski type inequalities for $q$-differentiable functions involving the special function introduced by R.K. Raina which depends on certain parameters. Our methodology involves Jackson's $q$-integral, the basic concepts of quantum calculus, and a generalization of a class of special functions used in the frame of convex sets and convex functions. As a main result, some quantum estimates for the aforementioned inequality are established and some cases involving the special hypergeometric and Mittag-Leffler functions have been studied and some known results are deduced.
\end{abstract}

Keywords: ostrowski inequality; quantum estimates; raina's function; generalized convexity

MSC: 26A51, 26A33, 26D07, 26D10

\section{Introduction}

Quantum calculus (also known as calculus with no limits) was initiated by Euler in the eighteenth century (1707-1783). In [1], F. J. Jackson started a systematic study of q-calculus and introduced the q-definite integrals. He also was the first to develop q-calculus in a systematic way. Some branches of mathematics and physics, such as number theory, orthogonal polynomials, combinatory, basic hypergeometric functions, mechanics, and quantum and relativity theory, have been enriched by the research work of various authors as T. Ernst [2,3], H. Gauchman [4], V. Kac and P. Cheung [5], and M.E.H. Ismail [6,7]. Also, certain famous integral inequalities have been studied in the frame of q-calculus [8,9].

The convexity of a function has played an important role as a tool in the development of inequalities. The relationship of this concept is always present in branches, such as functional analysis, harmonic analysis, specifically in interpolation theory, control theory and optimization, as is shown in the following works C.P. Niculescu [10], C. Bennett and R. Sharpley [11], N.A. Nguyen et. al. [12], S. Mititelu and S. Trenţă [13], S. Trenţă [14,15]. This property was defined by J.L.W.V. Jensen in the following works $[16,17]$ as follows. 
Definition 1. A function $f: I \subseteq \mathbb{R} \longrightarrow \mathbb{R}$ is said to be convex on $I$, if

$$
f\left((1-\imath) \ell_{1}+\imath \ell_{2}\right) \leq(1-\imath) f\left(\ell_{1}\right)+\imath f\left(\ell_{2}\right)
$$

holds for every $\ell_{1}, \ell_{2} \in I$ and $\imath \in[0,1]$.

It is well known that modern analysis directly or indirectly involves the applications of convexity. Due to its applications and significant importance, the concept of convexity has been extended and generalized in several directions. The concept of convexity and its variant forms have played a fundamental role in the development of various fields. Various types of generalized convexity have appeared in different research works, some of them modify the domain or range of the function, always maintaining the basic structure of a convex function. Among them are: s-convexity in the first and second sense [18], P-convexity [19], MT-convexity [20], and others [21-26].

The following inequality, named the Ostrowski inequality, is one of the most famous inequalities in the literature.

Theorem 1. Let $f: I \subseteq\left[0,+\infty\left[\longrightarrow \mathbb{R}\right.\right.$ be a differentiable function on $I$, such that $f^{\prime} \in L\left[\ell_{1}, \ell_{2}\right]$ and $\ell_{1}, \ell_{2} \in I$ with $\ell_{1}<\ell_{2}$. If $\left|f^{\prime}(x)\right| \leq M$, where $M$ is a real positive number, then the following inequality holds:

$$
\left|f(x)-\frac{1}{\ell_{2}-\ell_{1}} \int_{\ell_{1}}^{\ell_{2}} f(\imath) d \imath\right| \leq \frac{M}{\ell_{2}-\ell_{1}}\left[\frac{\left(x-\ell_{1}\right)^{2}+\left(\ell_{2}-x\right)^{2}}{2}\right] .
$$

The Ostrowski type inequality has remained a subject of great interest due to its wide applications in the field of mathematical analysis [27]. For other recent results that generalize, improve, and extend the inequality (1) through various class functions, for example, functions of bounded variation, Lipschitzian, monotone, absolutely continuous, convex, s-convex, $h$-convex, and $\left(m, h_{1}, h_{2}\right)$-convex among others, the studies [18,28-32] have appeared.

\section{Preliminaries}

Let $K$ be a non empty closed set in $\mathbb{R}^{n}$ and $\phi: K \rightarrow \mathbb{R}$ be a continuous function.

M.A. Noor, in [33], introduced a new class of non-convex functions, the so-called $\phi$-convex as follows:

Definition 2. The function $f: K \rightarrow \mathbb{R}$ on the $\phi$-convex set $K$ is said to be $\phi$-convex, if

$$
f\left(\ell_{1}+\imath e^{i \phi}\left(\ell_{2}-\ell_{1}\right)\right) \leq(1-\imath) f\left(\ell_{1}\right)+\imath f\left(\ell_{2}\right), \quad \forall \ell_{1}, \ell_{2} \in K, \imath \in[0,1] .
$$

The function $f$ is said to be $\phi$-concave if $(-f)$ is $\phi$-convex. Note that every convex function is $\phi$-convex but the converse does not hold in general.

R.K. Raina, in [34], introduced a class of functions defined by

$$
\mathcal{F}_{\rho, \lambda}^{\sigma}(z)=\mathcal{F}_{\rho, \lambda}^{\sigma(0), \sigma(1), \ldots}(z)=\sum_{k=0}^{+\infty} \frac{\sigma(k)}{\Gamma(\rho k+\lambda)} z^{k}
$$

where $\rho, \lambda>0,|z|<R$ and $\sigma=(\sigma(0), \ldots, \sigma(k), \ldots)$ is a bounded sequence of positive real numbers. Note that, if we take in (2) $\rho=1, \lambda=0$ and

$$
\sigma(k)=\frac{\left((\alpha)_{k}(\beta)_{k}\right)}{(\gamma)_{k}} \text { for } k=0,1,2, \ldots
$$


where $\alpha, \beta$ and $\gamma$ are parameters which can take arbitrary real or complex values (provided that $\gamma \neq 0,-1,-2, \ldots)$, and the symbol $(a)_{k}$ denotes the quantity

$$
(a)_{k}=\frac{\Gamma(a+k)}{\Gamma(a)}=a(a+1) \ldots(a+k-1), \quad k=0,1,2, \ldots,
$$

and we restrict its domain to $|z| \leq 1$ (with $z \in \mathbb{C}$ ), then we have the classical hypergeometric function, that is

$$
\mathcal{F}_{\rho, \lambda}^{\sigma}(z)=F(\alpha, \beta ; \gamma ; z)=\sum_{k=0}^{+\infty} \frac{(\alpha)_{k}(\beta)_{k}}{k !(\gamma)_{k}} z^{k}
$$

Also, if $\sigma=(1,1, \ldots)$ with $\rho=\alpha,(\operatorname{Re}(\alpha)>0), \lambda=1$ and if we restrict its domain to $z \in \mathbb{C}$ in (2) then we have the classical Mittag-Leffler function

$$
E_{\alpha}(z)=\sum_{k=0}^{+\infty} \frac{1}{\Gamma(1+\alpha k)} z^{k}
$$

Finally, let us recall the new class of sets and new class of functions involving Raina's function introduced by Vivas-Cortez et al. in [35]: the so-called generalized $\phi$-convex set and also the generalized $\phi$-convex function. The usefulness of this definition lies in the generality of the type of special function focused on. The $\sigma, \rho$ and $\lambda$ parameters determine the particular special function considered. Just as the definition used by Noor in [33] considers the $e^{i \phi}$ function as a parameter, in the framework of complex values, the following definition emulates it in the field of real numbers.

Definition 3. Let $\rho, \lambda>0$ and $\sigma=(\sigma(0), \ldots, \sigma(k), \ldots)$ be bounded sequences of positive real numbers. A non empty set $K$ is said to be a generalized $\phi$-convex set, if

$$
\ell_{1}+\imath \mathcal{F}_{\rho, \lambda}^{\sigma}\left(\ell_{2}-\ell_{1}\right) \in K, \quad \forall \ell_{1}, \ell_{2} \in K \text { and } \imath \in[0,1],
$$

where $\mathcal{F}_{\rho, \lambda}^{\sigma}(\cdot)$ is Raina's function.

Definition 4. Let $\rho, \lambda>0$ and $\sigma=(\sigma(0), \ldots, \sigma(k), \ldots)$ be bounded sequences of positive real numbers. If $a$ function $f: K \rightarrow \mathbb{R}$ satisfies the following inequality

$$
f\left(\ell_{1}+\imath \mathcal{F}_{\rho, \lambda}^{\sigma}\left(\ell_{2}-\ell_{1}\right)\right) \leq(1-\imath) f\left(\ell_{1}\right)+\imath f\left(\ell_{2}\right),
$$

for all $\imath \in[0,1]$ and $\ell_{1}, \ell_{2} \in K$, then $f$ is called generalized $\phi$-convex.

Remark 1. Taking $\mathcal{F}_{\rho, \lambda}^{\sigma}\left(\ell_{2}-\ell_{1}\right)=\ell_{2}-\ell_{1}>0$ in definition 4 , then we obtain definition 1. Also, under a suitable choice of $\mathcal{F}_{\rho, \lambda}^{\sigma}(\cdot)$, we find definition 2.

Recently several authors have utilized quantum calculus as a strong tool in establishing other inequalities, see $[5,36-40]$ and the references therein.

We recall now some concepts from quantum calculus. Let $I=\left[\ell_{1}, \ell_{2}\right] \subseteq \mathbb{R}$ be an interval and $0<q<1$ be a constant.

Definition 5. [39] Let $f: I \rightarrow \mathbb{R}$ be a continuous function and $x \in I$. Then the $q$-derivative of $f$ on $I$ at $x$ is defined as

$$
{ }_{\ell_{1}} \mathcal{D}_{q} f(x)=\frac{f(x)-f\left(q x+(1-q) \ell_{1}\right)}{(1-q)\left(x-\ell_{1}\right)}, x \neq \ell_{1}, \quad \ell_{1} \mathcal{D}_{q} f\left(\ell_{1}\right)=\lim _{x \rightarrow \ell_{1}} \ell_{1} \mathcal{D}_{q} f(x)
$$


We say that $f$ is $q$-differentiable on I provided ${ }_{\ell_{1}} \mathcal{D}_{q} f(x)$ exists for all $x \in I$. Note that if $\ell_{1}=0$ in (5), then $\ell_{1} \mathcal{D}_{q} f=\mathcal{D}_{q} f$, where $\mathcal{D}_{q}$ is the well-known $q$-derivative of the function $f(x)$ defined by

$$
\mathcal{D}_{q} f(x)=\frac{f(x)-f(q x)}{(1-q) x}
$$

Definition 6. [39] Let $f: I \rightarrow \mathbb{R}$ be a continuous function. Then the q-integral on I is defined by

$$
\int_{\ell_{1}}^{x} f(\imath){ }_{\ell_{1}} d_{q} \imath=(1-q)\left(x-\ell_{1}\right) \sum_{n=0}^{+\infty} q^{n} f\left(q^{n} x+\left(1-q^{n}\right) \ell_{1}\right) .
$$

for $x \in I$. Note that if $\ell_{1}=0$, then we have the classical $q$-integral, which is defined by

$$
\int_{0}^{x} f(\imath){ }_{0} d_{q} \imath=(1-q) x \sum_{n=0}^{+\infty} q^{n} f\left(q^{n} x\right)
$$

for $x \in[0,+\infty)$.

Theorem 2. [39] Assume that $f, g: I \rightarrow \mathbb{R}$ are continuous functions, $c \in \mathbb{R}$. Then, for $x \in I$, we have

$$
\begin{gathered}
\int_{\ell_{1}}^{x}[f(\imath)+g(\imath)] \ell_{1} d_{q} l=\int_{\ell_{1}}^{x} f(\imath)_{\ell_{1}} d_{q} l+\int_{\ell_{1}}^{x} g(\imath)_{\ell_{1}} d_{q} l ; \\
\int_{\ell_{1}}^{x}(c f)(\imath)_{\ell_{1}} d_{q} l=c \int_{\ell_{1}}^{x} f(l) \ell_{\ell_{1}} d_{q} l .
\end{gathered}
$$

Definition 7. [5] For any real number $\ell_{1}$,

$$
\left[\ell_{1}\right]_{q}=\frac{q^{\ell_{1}}-1}{q-1}
$$

is called the $q$-analogue of $\ell_{1}$. In particular, if $n \in \mathbb{Z}$, we denote

$$
[n]=\frac{q^{n}-1}{q-1}=q^{n-1}+\cdots+q+1 .
$$

Definition 8. [5] If $n \in \mathbb{Z}$, the $q$-analogue of $\left(x-\ell_{1}\right)^{n}$ is the polynomial

$$
\left(x-\ell_{1}\right)_{q}^{n}= \begin{cases}1, & n=0 \\ \left(x-\ell_{1}\right)\left(x-q \ell_{1}\right) \cdots\left(x-q^{n-1} \ell_{1}\right), & n \geq 1 .\end{cases}
$$

Definition 9. [5] For any $t, s>0$,

$$
\beta_{q}(t, s)=\int_{0}^{1}{ }^{t-1}(1-q \imath)_{q}^{s-1}{ }_{0} d_{q} \imath
$$

is called the q-Beta function. Note that

$$
\beta_{q}(t, 1)=\int_{0}^{1}{ }^{t-1}{ }_{0} d_{q} l=\frac{1}{[t]},
$$

where $[t]$ is the q-analogue of $t$.

Noor M. A. et al. in [31], established the following $q$-integral identity. 
Lemma 1. Let $f:\left[\ell_{1}, \ell_{2}\right] \subseteq \mathbb{R} \rightarrow \mathbb{R}$ be a $q$-differentiable function on $\left(\ell_{1}, \ell_{2}\right)$ with ${ }_{\ell_{1}} \mathcal{D}_{q} f$ be continuous and integrable on $\left[\ell_{1}, \ell_{2}\right]$, where $0<q<1$. Then the following identity holds:

$$
\begin{gathered}
f(x)-\frac{1}{\ell_{2}-\ell_{1}} \int_{\ell_{1}}^{\ell_{2}} f(\imath)_{\ell_{1}} d_{q} \imath=\frac{q\left(x-\ell_{1}\right)^{2}}{\ell_{2}-\ell_{1}} \int_{0}^{1} \imath_{\ell_{1}} \mathcal{D}_{q} f\left(\imath x+(1-\imath) \ell_{1}\right)_{0} d_{q} \imath \\
+\frac{q\left(\ell_{2}-x\right)^{2}}{\ell_{2}-\ell_{1}} \int_{0}^{1} \imath_{\ell_{1}} \mathcal{D}_{q} f\left(\imath x+(1-\imath) \ell_{2}\right)_{0} d_{q} \imath .
\end{gathered}
$$

Motivated by the above literatures, the paper is organized as follows: The Main Results section is divided in two subsections; the first is a study about quantum estimates for Ostrowski inequalities using the generalized class of special functions defined by Raina R.K., and the second subsection presents some Ostrowski type inequalities for certain special functions derived from the previous results. We will use an identity for $q$-differentiable functions involving Raina's function and applying this identity, we develop some quantum estimates of Ostrowski type inequalities for the generalized $\phi$-convex function. Various special cases will be obtained.

\section{Main Results}

This section is subdivided in two subsections. The first is about some quantum estimates for Ostrowski inequalities using the class of functions defined by Raina R.K., and in the second subsection are results about these quantum estimates for the hypergeometric function and the Mittag-Leffler function.

\subsection{Quantum Estimates for Ostrowski Inequality}

We denote $O=\left[\ell_{1}, \ell_{2}\right]$, for $\ell_{1}<\ell_{2}$, where $\rho, \lambda>0$ and $\sigma=(\sigma(0), \ldots, \sigma(k), \ldots)$ is a bounded sequence of positive real numbers. Also, for convenience we denote $d_{q} l$ for ${ }_{0} d_{q} l$, where $0<q<1$.

We first derive a new quantum integral identity for $q$-differentiable functions involving Raina's function.

Lemma 2. Let $f: O \rightarrow \mathbb{R}$ be a q-differentiable function on $O^{\circ}$ with ${ }_{\ell_{1}} \mathcal{D}_{q} f$ considered continuous and integrable on $O$. Then the following identity holds:

$$
\begin{gathered}
T_{f}\left(x, \ell_{1}, \ell_{2} ; q\right)=\frac{q\left[\mathcal{F}_{\rho, \lambda}^{\sigma}\left(x-\ell_{1}\right)\right]^{2}}{\mathcal{F}_{\rho, \lambda}^{\sigma}\left(x-\ell_{1}\right)+\mathcal{F}_{\rho, \lambda}^{\sigma}\left(\ell_{2}-x\right)} \int_{0}^{1} \imath_{\ell_{1}} \mathcal{D}_{q} f\left(\ell_{1}+\imath \mathcal{F}_{\rho, \lambda}^{\sigma}\left(x-\ell_{1}\right)\right) d_{q} l \\
+\frac{q\left[\mathcal{F}_{\rho, \lambda}^{\sigma}\left(\ell_{2}-x\right)\right]^{2}}{\mathcal{F}_{\rho, \lambda}^{\sigma}\left(x-\ell_{1}\right)+\mathcal{F}_{\rho, \lambda}^{\sigma}\left(\ell_{2}-x\right)} \int_{0}^{1} \imath_{\ell_{1}} \mathcal{D}_{q} f\left(\ell_{2}+\imath \mathcal{F}_{\rho, \lambda}^{\sigma}\left(x-\ell_{2}\right)\right) d_{q} l
\end{gathered}
$$

where

$$
T_{f}\left(x, \ell_{1}, \ell_{2} ; q\right)=f(x)-\frac{1}{\mathcal{F}_{\rho, \lambda}^{\sigma}\left(x-\ell_{1}\right)+\mathcal{F}_{\rho, \lambda}^{\sigma}\left(\ell_{2}-x\right)} \int_{\ell_{1}}^{\ell_{1}+\mathcal{F}_{\rho, \lambda}^{\sigma}\left(\ell_{2}-\ell_{1}\right)} f(\iota) \ell_{1} d_{q} \imath
$$

Proof. Let denote, respectively,

$$
T_{f}^{(1)}\left(x, \ell_{1}, \ell_{2} ; q\right)=\frac{q\left[\mathcal{F}_{\rho, \lambda}^{\sigma}\left(x-\ell_{1}\right)\right]^{2}}{\mathcal{F}_{\rho, \lambda}^{\sigma}\left(x-\ell_{1}\right)+\mathcal{F}_{\rho, \lambda}^{\sigma}\left(\ell_{2}-x\right)} \int_{0}^{1}{ }^{1}{ }_{\ell_{1}} \mathcal{D}_{q} f\left(\ell_{1}+\imath \mathcal{F}_{\rho, \lambda}^{\sigma}\left(x-\ell_{1}\right)\right) d_{q} l
$$

and

$$
T_{f}^{(2)}\left(x, \ell_{1}, \ell_{2} ; q\right)=\frac{q\left[\mathcal{F}_{\rho, \lambda}^{\sigma}\left(\ell_{2}-x\right)\right]^{2}}{\mathcal{F}_{\rho, \lambda}^{\sigma}\left(x-\ell_{1}\right)+\mathcal{F}_{\rho, \lambda}^{\sigma}\left(\ell_{2}-x\right)} \int_{0}^{1}{ }^{1}{ }_{\ell_{1}} \mathcal{D}_{q} f\left(\ell_{2}+\imath \mathcal{F}_{\rho, \lambda}^{\sigma}\left(x-\ell_{2}\right)\right) d_{q} l .
$$


Then, we have

$$
T_{f}\left(x, \ell_{1}, \ell_{2} ; q\right)=T_{f}^{(1)}\left(x, \ell_{1}, \ell_{2} ; q\right)+T_{f}^{(2)}\left(x, \ell_{1}, \ell_{2} ; q\right) .
$$

Using Definitions 5 and 6, we have

$$
\begin{aligned}
& T_{f}^{(1)}\left(x, \ell_{1}, \ell_{2} ; q\right)=\frac{q\left[\mathcal{F}_{\rho, \lambda}^{\sigma}\left(x-\ell_{1}\right)\right]^{2}}{\mathcal{F}_{\rho, \lambda}^{\sigma}\left(x-\ell_{1}\right)+\mathcal{F}_{\rho, \lambda}^{\sigma}\left(\ell_{2}-x\right)} \\
& \times \int_{0}^{1} \imath \frac{f\left(\ell_{1}+\imath \mathcal{F}_{\rho, \lambda}^{\sigma}\left(x-\ell_{1}\right)\right)-f\left(\ell_{1}+q \imath \mathcal{F}_{\rho, \lambda}^{\sigma}\left(x-\ell_{1}\right)\right)}{(1-q) \imath \mathcal{F}_{\rho, \lambda}^{\sigma}\left(x-\ell_{1}\right)} d_{q} l \\
& =\frac{\mathcal{F}_{\rho, \lambda}^{\sigma}\left(x-\ell_{1}\right)}{\mathcal{F}_{\rho, \lambda}^{\sigma}\left(x-\ell_{1}\right)+\mathcal{F}_{\rho, \lambda}^{\sigma}\left(\ell_{2}-x\right)} \\
& \times\left[\sum_{n=0}^{+\infty} q^{n+1} f\left(\ell_{1}+q^{n} \mathcal{F}_{\rho, \lambda}^{\sigma}\left(x-\ell_{1}\right)\right)-\sum_{n=0}^{+\infty} q^{n+1} f\left(\ell_{1}+q^{n+1} \mathcal{F}_{\rho, \lambda}^{\sigma}\left(x-\ell_{1}\right)\right)\right] \\
& =\frac{\mathcal{F}_{\rho_{, \lambda}}^{\sigma}\left(x-\ell_{1}\right)}{\mathcal{F}_{\rho, \lambda}^{\sigma}\left(x-\ell_{1}\right)+\mathcal{F}_{\rho, \lambda}^{\sigma}\left(\ell_{2}-x\right)} f(x)-\frac{1}{\mathcal{F}_{\rho, \lambda}^{\sigma}\left(x-\ell_{1}\right)+\mathcal{F}_{\rho, \lambda}^{\sigma}\left(\ell_{2}-x\right)} \int_{\ell_{1}}^{x} f(\imath) d_{q} \imath .
\end{aligned}
$$

Similarly

$$
\begin{aligned}
T_{f}^{(2)}\left(x, \ell_{1}, \ell_{2} ; q\right)=\frac{\mathcal{F}_{\rho, \lambda}^{\sigma}\left(\ell_{2}-x\right)}{\mathcal{F}_{\rho, \lambda}^{\sigma}\left(x-\ell_{1}\right)+\mathcal{F}_{\rho, \lambda}^{\sigma}\left(\ell_{2}-x\right)} f(x) & \\
& -\frac{1}{\mathcal{F}_{\rho, \lambda}^{\sigma}\left(x-\ell_{1}\right)+\mathcal{F}_{\rho, \lambda}^{\sigma}\left(\ell_{2}-x\right)} \int_{x}^{\ell_{1}+\mathcal{F}_{\rho, \lambda}^{\sigma}\left(\ell_{2}-\ell_{1}\right)} f(\imath) d_{q} l .
\end{aligned}
$$

Adding (7) and (8), we get (6).

The proof is complete.

Remark 2. Taking $q \rightarrow 1^{-}$in Lemma 2, we obtain the following new identity:

$$
\begin{gathered}
T_{f}\left(x, \ell_{1}, \ell_{2}\right)=\frac{\left[\mathcal{F}_{\rho, \lambda}^{\sigma}\left(x-\ell_{1}\right)\right]^{2}}{\mathcal{F}_{\rho, \lambda}^{\sigma}\left(x-\ell_{1}\right)+\mathcal{F}_{\rho, \lambda}^{\sigma}\left(\ell_{2}-x\right)} \int_{0}^{1} \imath f^{\prime}\left(\ell_{1}+\imath \mathcal{F}_{\rho, \lambda}^{\sigma}\left(x-\ell_{1}\right)\right) d \imath \\
+\frac{\left[\mathcal{F}_{\rho, \lambda}^{\sigma}\left(\ell_{2}-x\right)\right]^{2}}{\mathcal{F}_{\rho, \lambda}^{\sigma}\left(x-\ell_{1}\right)+\mathcal{F}_{\rho, \lambda}^{\sigma}\left(\ell_{2}-x\right)} \int_{0}^{1} \imath f^{\prime}\left(\ell_{2}+\imath \mathcal{F}_{\rho, \lambda}^{\sigma}\left(x-\ell_{2}\right)\right) d \imath,
\end{gathered}
$$

where

$$
T_{f}\left(x, \ell_{1}, \ell_{2}\right)=f(x)-\frac{1}{\mathcal{F}_{\rho, \lambda}^{\sigma}\left(x-\ell_{1}\right)+\mathcal{F}_{\rho, \lambda}^{\sigma}\left(\ell_{2}-x\right)} \int_{\ell_{1}}^{\ell_{1}+\mathcal{F}_{\rho, \lambda}^{\sigma}\left(\ell_{2}-\ell_{1}\right)} f(\imath) d \imath .
$$

Remark 3. Letting $\rho=1, \lambda=0$ and $\sigma=(0,1,0,0, \cdots)$ in Lemma 2 we have $\mathcal{F}_{\rho, \lambda}^{\sigma}(y-x)=y-x$ for all $x, y \in O$, and so we get Lemma 1 .

Now, applying Lemma 2, we establish some quantum estimates of Ostrowski type inequalities for generalized $\phi$-convex functions. 
Theorem 3. Let $f: O \rightarrow \mathbb{R}$ be a q-differentiable function on $O^{\circ}$ with ${ }_{\ell_{1}} \mathcal{D}_{q} f$ considered continuous and integrable on $O$. If $\left|\ell_{1} \mathcal{D}_{q} f\right|^{r}$ is generalized as $\phi$-convex on $O$ for $r>1$ and $\frac{1}{p}+\frac{1}{r}=1$, then the following inequality holds:

$$
\begin{aligned}
\left|T_{f}\left(x, \ell_{1}, \ell_{2} ; q\right)\right| \leq \frac{q}{\sqrt[p]{[p+1]}\left(\mathcal{F}_{\rho, \lambda}^{\sigma}\left(x-\ell_{1}\right)+\mathcal{F}_{\rho, \lambda}^{\sigma}\left(\ell_{2}-x\right)\right)} \times \\
\left\{\left[\mathcal{F}_{\rho, \lambda}^{\sigma}\left(x-\ell_{1}\right)\right]^{2} \sqrt[r]{\frac{\left.\left.q\right|_{\ell_{1}} \mathcal{D}_{q} f\left(\ell_{1}\right)\right|^{r}+\left|\ell_{1} \mathcal{D}_{q} f(x)\right|^{r}}{1+q}}\right. \\
\left.+\left[\mathcal{F}_{\rho, \lambda}^{\sigma}\left(\ell_{2}-x\right)\right]^{2} \sqrt[r]{\frac{\left.\left.q\right|_{\ell_{1}} \mathcal{D}_{q} f\left(\ell_{2}\right)\right|^{r}+\left.\left.\right|_{\ell_{1}} \mathcal{D}_{q} f(x)\right|^{r}}{1+q}}\right\},
\end{aligned}
$$

where $[p+1]$ is the $q$-analogue of $p+1$.

Proof. Using Lemma 2, Hölder's inequality and the fact that $\left.{\mid \ell_{1}}_{\mathcal{D}_{q}}\right|^{r}$ is generalized $\phi$-convex function, we have

$$
\begin{aligned}
& \left|T_{f}\left(x, \ell_{1}, \ell_{2} ; q\right)\right| \\
& \leq\left.\frac{q\left[\mathcal{F}_{\rho, \lambda}^{\sigma}\left(x-\ell_{1}\right)\right]^{2}}{\mathcal{F}_{\rho, \lambda}^{\sigma}\left(x-\ell_{1}\right)+\mathcal{F}_{\rho, \lambda}^{\sigma}\left(\ell_{2}-x\right)} \int_{0}^{1} \imath\right|_{\ell_{1}} \mathcal{D}_{q} f\left(\ell_{1}+\imath \mathcal{F}_{\rho, \lambda}^{\sigma}\left(x-\ell_{1}\right)\right) \mid d_{q} \imath \\
& +\left.\frac{q\left[\mathcal{F}_{\rho, \lambda}^{\sigma}\left(\ell_{2}-x\right)\right]^{2}}{\mathcal{F}_{\rho, \lambda}^{\sigma}\left(x-\ell_{1}\right)+\mathcal{F}_{\rho, \lambda}^{\sigma}\left(\ell_{2}-x\right)} \int_{0}^{1} \imath\right|_{\ell_{1}} \mathcal{D}_{q} f\left(\ell_{2}+\imath \mathcal{F}_{\rho, \lambda}^{\sigma}\left(x-\ell_{2}\right)\right) \mid d_{q} l \\
& \leq \frac{q\left[\mathcal{F}_{\rho, \lambda}^{\sigma}\left(x-\ell_{1}\right)\right]^{2}}{\mathcal{F}_{\rho, \lambda}^{\sigma}\left(x-\ell_{1}\right)+\mathcal{F}_{\rho, \lambda}^{\sigma}\left(\ell_{2}-x\right)}\left(\int_{0}^{1}{ }^{p}{ }^{p} d_{q} \imath\right)^{\frac{1}{p}}\left(\int_{0}^{1}\left|{ }_{\ell_{1}} \mathcal{D}_{q} f\left(\ell_{1}+\imath \mathcal{F}_{\rho, \lambda}^{\sigma}\left(x-\ell_{1}\right)\right)\right|^{r} d_{q} \imath\right)^{\frac{1}{r}} \\
& +\frac{q\left[\mathcal{F}_{\rho, \lambda}^{\sigma}\left(\ell_{2}-x\right)\right]^{2}}{\mathcal{F}_{\rho, \lambda}^{\sigma}\left(x-\ell_{1}\right)+\mathcal{F}_{\rho, \lambda}^{\sigma}\left(\ell_{2}-x\right)}\left(\int_{0}^{1}{ }^{1}{ }^{p} d_{q}\right)^{\frac{1}{p}} \times \\
& \left(\int_{0}^{1}\left|l_{1} \mathcal{D}_{q} f\left(\ell_{2}+\imath \mathcal{F}_{\rho, \lambda}^{\sigma}\left(x-\ell_{2}\right)\right)\right|^{r} d_{q}\right)^{\frac{1}{r}} \\
& \leq \frac{q\left[\mathcal{F}_{\rho, \lambda}^{\sigma}\left(x-\ell_{1}\right)\right]^{2}}{\mathcal{F}_{\rho, \lambda}^{\sigma}\left(x-\ell_{1}\right)+\mathcal{F}_{\rho, \lambda}^{\sigma}\left(\ell_{2}-x\right)}\left(\int_{0}^{1}{ }^{p} d_{q} \imath\right)^{\frac{1}{p}} \times \\
& \left(\int_{0}^{1}\left[\left.\left.(1-\imath)\right|_{\ell_{1}} \mathcal{D}_{q} f\left(\ell_{1}\right)\right|^{r}+\left.\left.\imath\right|_{\ell_{1}} \mathcal{D}_{q} f(x)\right|^{r}\right] d_{q} \imath\right)^{\frac{1}{r}} \\
& +\frac{q\left[\mathcal{F}_{\rho, \lambda}^{\sigma}\left(\ell_{2}-x\right)\right]^{2}}{\mathcal{F}_{\rho, \lambda}^{\sigma}\left(x-\ell_{1}\right)+\mathcal{F}_{\rho, \lambda}^{\sigma}\left(\ell_{2}-x\right)}\left(\int_{0}^{1} \imath^{p} d_{q}\right)^{\frac{1}{p}} \times \\
& \left(\int_{0}^{1}\left[\left.\left.(1-\imath)\right|_{\ell_{1}} \mathcal{D}_{q} f\left(\ell_{2}\right)\right|^{r}+\left.\left.\imath\right|_{\ell_{1}} \mathcal{D}_{q} f(x)\right|^{r}\right] d_{q} \imath\right)^{\frac{1}{r}}
\end{aligned}
$$




$$
\begin{aligned}
=\frac{q}{\sqrt[p]{[p+1]}} \times \frac{1}{\mathcal{F}_{\rho, \lambda}^{\sigma}\left(x-\ell_{1}\right)+\mathcal{F}_{\rho, \lambda}^{\sigma}\left(\ell_{2}-x\right)} \times & \\
\left\{\left[\mathcal{F}_{\rho, \lambda}^{\sigma}\left(x-\ell_{1}\right)\right]^{2} \sqrt[r]{\frac{\left.\left.q\right|_{\ell_{1}} \mathcal{D}_{q} f\left(\ell_{1}\right)\right|^{r}+\left.||_{\ell_{1}} \mathcal{D}_{q} f(x)\right|^{r}}{1+q}}\right. & \\
& \left.+\left[\mathcal{F}_{\rho, \lambda}^{\sigma}\left(\ell_{2}-x\right)\right]^{2} \sqrt[r]{\frac{\left.\left.q\right|_{\ell_{1}} \mathcal{D}_{q} f\left(\ell_{2}\right)\right|^{r}+\left.\left.\right|_{\ell_{1}} \mathcal{D}_{q} f(x)\right|^{r}}{1+q}}\right\} .
\end{aligned}
$$

The proof is complete.

Corollary 1. Taking the limit when $q \rightarrow 1^{-}$in Theorem 3, we obtain

$$
\begin{aligned}
&\left|T_{f}\left(x, \ell_{1}, \ell_{2}\right)\right| \leq \frac{1}{\sqrt[p]{p+1}\left(\mathcal{F}_{\rho, \lambda}^{\sigma}\left(x-\ell_{1}\right)+\mathcal{F}_{\rho, \lambda}^{\sigma}\left(\ell_{2}-x\right)\right)} \times \\
&\left\{\left[\mathcal{F}_{\rho, \lambda}^{\sigma}\left(x-\ell_{1}\right)\right]^{2} \sqrt[r]{\frac{\left|f^{\prime}\left(\ell_{1}\right)\right|^{r}+\left|f^{\prime}(x)\right|^{r}}{2}}+\left[\mathcal{F}_{\rho, \lambda}^{\sigma}\left(\ell_{2}-x\right)\right]^{2} \sqrt[r]{\frac{\left|f^{\prime}\left(\ell_{2}\right)\right|^{r}+\left|f^{\prime}(x)\right|^{r}}{2}}\right\} .
\end{aligned}
$$

Corollary 2. Additionally, if $\left.\right|_{\ell_{1}} \mathcal{D}_{q} f \mid \leq M$ for some $M>0$ in Theorem 3, then the following inequality holds

$$
\left|T_{f}\left(x, \ell_{1}, \ell_{2} ; q\right)\right| \leq \frac{q M}{\sqrt[p]{[p+1]}}\left[\frac{\left[\mathcal{F}_{\rho, \lambda}^{\sigma}\left(x-\ell_{1}\right)\right]^{2}+\left[\mathcal{F}_{\rho, \lambda}^{\sigma}\left(\ell_{2}-x\right)\right]^{2}}{\mathcal{F}_{\rho, \lambda}^{\sigma}\left(x-\ell_{1}\right)+\mathcal{F}_{\rho, \lambda}^{\sigma}\left(\ell_{2}-x\right)}\right] .
$$

Remark 4. Letting $\rho=1, \lambda=0$ and $\sigma=(0,1,0,0, \cdots)$ in the Raina's function then we have $\mathcal{F}_{\rho, \lambda}^{\sigma}(y-x)=$ $y-x$ for all $x, y \in O$, so from Corollary 2, we obtain Theorem 3.2 in [31].

Theorem 4. Let $f: O \rightarrow \mathbb{R}$ be a q-differentiable function on $O^{\circ}$ with ${ }_{\ell_{1}} \mathcal{D}_{q} f$ considered continuous and integrable on $O$. If $\left.\left.\right|_{\ell_{1}} \mathcal{D}_{q} f\right|^{r}$ is generalized as $\phi$-convex on $O$, then for $r \geq 1$, the following inequality holds:

$$
\begin{aligned}
\left|T_{f}\left(x, \ell_{1}, \ell_{2} ; q\right)\right| \leq & \frac{q}{(1+q) \sqrt[r]{1+q+q^{2}}\left(\mathcal{F}_{\rho, \lambda}^{\sigma}\left(x-\ell_{1}\right)+\mathcal{F}_{\rho, \lambda}^{\sigma}\left(\ell_{2}-x\right)\right)} \times \\
& \left\{\left[\mathcal{F}_{\rho, \lambda}^{\sigma}\left(x-\ell_{1}\right)\right]^{2} \sqrt[r]{q^{2}\left|\ell_{1} \mathcal{D}_{q} f\left(\ell_{1}\right)\right|^{r}+\left.\left.(1+q)\right|_{\ell_{1}} \mathcal{D}_{q} f(x)\right|^{r}}\right. \\
& \left.+\left[\mathcal{F}_{\rho, \lambda}^{\sigma}\left(\ell_{2}-x\right)\right]^{2} \sqrt[r]{q^{2}\left|{ }_{\ell_{1}} \mathcal{D}_{q} f\left(\ell_{2}\right)\right|^{r}+(1+q)\left|\ell_{1} \mathcal{D}_{q} f(x)\right|^{r}}\right\}
\end{aligned}
$$

Proof. Using Lemma 2, the well-known power mean inequality and the fact that $\left|\ell_{1} \mathcal{D}_{q} f\right|^{r}$ is a generalized $\phi$-convex function, we have

$$
\begin{aligned}
& \left|T_{f}\left(x, \ell_{1}, \ell_{2} ; q\right)\right| \begin{array}{r}
q\left[\mathcal{F}_{\rho, \lambda}^{\sigma}\left(x-\ell_{1}\right)\right]^{2} \\
\leq\left.\frac{\mathcal{F}_{\rho, \lambda}^{\sigma}\left(x-\ell_{1}\right)+\mathcal{F}_{\rho, \lambda}^{\sigma}\left(\ell_{2}-x\right)}{\int_{0}}{ }^{1} \imath\right|_{\ell_{1}} \mathcal{D}_{q} f\left(\ell_{1}+\imath \mathcal{F}_{\rho, \lambda}^{\sigma}\left(x-\ell_{1}\right)\right) \mid d_{q} l
\end{array} \\
& \quad+\left.\frac{q\left[\mathcal{F}_{\rho, \lambda}^{\sigma}\left(\ell_{2}-x\right)\right]^{2}}{\mathcal{F}_{\rho, \lambda}^{\sigma}\left(x-\ell_{1}\right)+\mathcal{F}_{\rho, \lambda}^{\sigma}\left(\ell_{2}-x\right)} \int_{0}^{1} \imath\right|_{\ell_{1}} \mathcal{D}_{q} f\left(\ell_{2}+\imath \mathcal{F}_{\rho, \lambda}^{\sigma}\left(x-\ell_{2}\right)\right) \mid d_{q} l \\
& \leq \frac{q\left[\mathcal{F}_{\rho, \lambda}^{\sigma}\left(x-\ell_{1}\right)\right]^{2}}{\mathcal{F}_{\rho, \lambda}^{\sigma}\left(x-\ell_{1}\right)+\mathcal{F}_{\rho, \lambda}^{\sigma}\left(\ell_{2}-x\right)}\left(\int_{0}^{1} \imath d_{q} \imath\right)^{1-\frac{1}{r}}\left(\left.\left.\int_{0}^{1} \imath\right|_{\ell_{1}} \mathcal{D}_{q} f\left(\ell_{1}+\imath \mathcal{F}_{\rho, \lambda}^{\sigma}\left(x-\ell_{1}\right)\right)\right|^{r} d_{q} \imath\right)^{\frac{1}{r}}
\end{aligned}
$$




$$
\begin{aligned}
& +\frac{q\left[\mathcal{F}_{\rho, \lambda}^{\sigma}\left(\ell_{2}-x\right)\right]^{2}}{\mathcal{F}_{\rho, \lambda}^{\sigma}\left(x-\ell_{1}\right)+\mathcal{F}_{\rho, \lambda}^{\sigma}\left(\ell_{2}-x\right)}\left(\int_{0}^{1} \imath d_{q} \iota\right)^{1-\frac{1}{r}}\left(\left.\left.\int_{0}^{1} \imath\right|_{\ell_{1}} \mathcal{D}_{q} f\left(\ell_{2}+\imath \mathcal{F}_{\rho, \lambda}^{\sigma}\left(x-\ell_{2}\right)\right)\right|^{r} d_{q} \iota\right)^{\frac{1}{r}} \\
& \leq \frac{q\left[\mathcal{F}_{\rho, \lambda}^{\sigma}\left(x-\ell_{1}\right)\right]^{2}}{\mathcal{F}_{\rho, \lambda}^{\sigma}\left(x-\ell_{1}\right)+\mathcal{F}_{\rho, \lambda}^{\sigma}\left(\ell_{2}-x\right)}\left(\int_{0}^{1} \imath d_{q} \imath\right)^{1-\frac{1}{r}} \times \\
& \left(\int_{0}^{1} \imath\left[\left.\left.(1-\imath)\right|_{\ell_{1}} \mathcal{D}_{q} f\left(\ell_{1}\right)\right|^{r}+\left.\left.\imath\right|_{\ell_{1}} \mathcal{D}_{q} f(x)\right|^{r}\right] d_{q} \imath\right)^{\frac{1}{r}} \\
& +\frac{q\left[\mathcal{F}_{\rho, \lambda}^{\sigma}\left(\ell_{2}-x\right)\right]^{2}}{\mathcal{F}_{\rho, \lambda}^{\sigma}\left(x-\ell_{1}\right)+\mathcal{F}_{\rho, \lambda}^{\sigma}\left(\ell_{2}-x\right)}\left(\int_{0}^{1} \imath d_{q} \imath\right)^{1-\frac{1}{r}} \times \\
& \left(\int_{0}^{1} \imath\left[\left.\left.(1-\imath)\right|_{\ell_{1}} \mathcal{D}_{q} f\left(\ell_{2}\right)\right|^{r}+\left.\left.\imath\right|_{\ell_{1}} \mathcal{D}_{q} f(x)\right|^{r}\right] d_{q} \imath\right)^{\frac{1}{r}} \\
& =\frac{q}{(1+q) \sqrt[r]{1+q+q^{2}}} \times \frac{1}{\mathcal{F}_{\rho, \lambda}^{\sigma}\left(x-\ell_{1}\right)+\mathcal{F}_{\rho, \lambda}^{\sigma}\left(\ell_{2}-x\right)} \\
& \left\{\left[\mathcal{F}_{\rho, \lambda}^{\sigma}\left(x-\ell_{1}\right)\right]^{2} \sqrt[r]{q^{2}\left|{ }_{\ell_{1}} \mathcal{D}_{q} f\left(\ell_{1}\right)\right|^{r}+\left.\left.(1+q)\right|_{\ell_{1}} \mathcal{D}_{q} f(x)\right|^{r}}\right. \\
& \left.+\left[\mathcal{F}_{\rho, \lambda}^{\sigma}\left(\ell_{2}-x\right)\right] \sqrt[r]{\left.\left.q^{2}\right|_{\ell_{1}} \mathcal{D}_{q} f\left(\ell_{2}\right)\right|^{r}+(1+q)\left|\ell_{1} \mathcal{D}_{q} f(x)\right|^{r}}\right\} .
\end{aligned}
$$

The proof is complete.

Corollary 3. Taking the limit when $q \rightarrow 1^{-}$in Theorem 4, we find

$$
\begin{aligned}
& \left|T_{f}\left(x, \ell_{1}, \ell_{2}\right)\right| \leq \frac{1}{2\left[\mathcal{F}_{\rho, \lambda}^{\sigma}\left(x-\ell_{1}\right)+\mathcal{F}_{\rho, \lambda}^{\sigma}\left(\ell_{2}-x\right)\right]} \times \\
& \quad\left\{\left[\mathcal{F}_{\rho, \lambda}^{\sigma}\left(x-\ell_{1}\right)\right]^{2} \sqrt[r]{\frac{\left|f^{\prime}\left(\ell_{1}\right)\right|^{r}+2\left|f^{\prime}(x)\right|^{r}}{3}}+\left[\mathcal{F}_{\rho, \lambda}^{\sigma}\left(\ell_{2}-x\right)\right]^{2} \sqrt[r]{\frac{\left|f^{\prime}\left(\ell_{2}\right)\right|^{r}+2\left|f^{\prime}(x)\right|^{r}}{3}}\right\} .
\end{aligned}
$$

Corollary 4. Additionally, if $\left.\right|_{\ell_{1}} \mathcal{D}_{q} f \mid \leq M$ for some $M>0$ in Theorem 4, then we obtain

$$
\left|T_{f}\left(x, \ell_{1}, \ell_{2} ; q\right)\right| \leq \frac{q M}{1+q}\left[\frac{\left[\mathcal{F}_{\rho, \lambda}^{\sigma}\left(x-\ell_{1}\right)\right]^{2}+\left[\mathcal{F}_{\rho, \lambda}^{\sigma}\left(\ell_{2}-x\right)\right]^{2}}{\mathcal{F}_{\rho, \lambda}^{\sigma}\left(x-\ell_{1}\right)+\mathcal{F}_{\rho, \lambda}^{\sigma}\left(\ell_{2}-x\right)}\right] .
$$

Remark 5. Letting $\rho=1, \lambda=0$ and $\sigma=(0,1,0,0, \cdots)$ in Raina's function then we have $\mathcal{F}_{\rho, \lambda}^{\sigma}(y-x)=$ $y-x$ for all $x, y \in O$, so from Corollary 4 we obtain Theorem 3.1 in [31]. 
Theorem 5. Let $f: O \rightarrow \mathbb{R}$ be a q-differentiable function on $O^{\circ}$ with ${ }_{\ell_{1}} \mathcal{D}_{q} f$ considered continuous and integrable on $O$. If $\left|\ell_{1} \mathcal{D}_{q} f\right|^{r}$ is generalized as $\phi$-convex on $O$, then for $r \geq 1$, the following inequality holds:

$$
\begin{aligned}
\left|T_{f}\left(x, \ell_{1}, \ell_{2} ; q\right)\right| \leq \frac{q}{\mathcal{F}_{\rho, \lambda}^{\sigma}\left(x-\ell_{1}\right)+\mathcal{F}_{\rho, \lambda}^{\sigma}\left(\ell_{2}-x\right)}\left(\frac{1-q}{1-q^{r+2}}\right)^{1 / r} \times \\
\left\{\left[\mathcal{F}_{\rho, \lambda}^{\sigma}\left(x-\ell_{1}\right)\right]^{2} \sqrt[r]{\left.\left.\frac{q^{r}(q-1)}{q^{r+1}-1}\right|_{\ell_{1}} \mathcal{D}_{q} f\left(\ell_{1}\right)\right|^{r}+\left.\left.\right|_{\ell_{1}} \mathcal{D}_{q} f(x)\right|^{r}}\right. \\
\left.+\left[\mathcal{F}_{\rho, \lambda}^{\sigma}\left(\ell_{2}-x\right)\right]^{2} \sqrt[r]{\left.\left.\frac{q^{r}(q-1)}{q^{r+1}-1}\right|_{\ell_{1}} \mathcal{D}_{q} f\left(\ell_{2}\right)\right|^{r}+\left.\left.\right|_{\ell_{1}} \mathcal{D}_{q} f(x)\right|^{r}}\right\} .
\end{aligned}
$$

Proof. Using Lemma 2, the well-known power mean inequality and the fact that $\left|{ }_{\ell_{1}} \mathcal{D}_{q} f\right|^{r}$ is a generalized $\phi$-convex function, we have

$$
\begin{aligned}
& \left|T_{f}\left(x, \ell_{1}, \ell_{2} ; q\right)\right| \\
& \leq\left.\frac{q\left[\mathcal{F}_{\rho, \lambda}^{\sigma}\left(x-\ell_{1}\right)\right]^{2}}{\mathcal{F}_{\rho, \lambda}^{\sigma}\left(x-\ell_{1}\right)+\mathcal{F}_{\rho, \lambda}^{\sigma}\left(\ell_{2}-x\right)} \int_{0}^{1} \imath\right|_{\ell_{1}} \mathcal{D}_{q} f\left(\ell_{1}+\imath \mathcal{F}_{\rho, \lambda}^{\sigma}\left(x-\ell_{1}\right)\right) \mid d_{q} l \\
& +\frac{q\left[\mathcal{F}_{\rho, \lambda}^{\sigma}\left(\ell_{2}-x\right)\right]^{2}}{\mathcal{F}_{\rho, \lambda}^{\sigma}\left(x-\ell_{1}\right)+\mathcal{F}_{\rho, \lambda}^{\sigma}\left(\ell_{2}-x\right)} \int_{0}^{1} \imath_{\ell_{1}} \mathcal{D}_{q} f\left(\ell_{2}+\imath \mathcal{F}_{\rho, \lambda}^{\sigma}\left(x-\ell_{2}\right)\right) \mid d_{q} l \\
& \leq \frac{q\left[\mathcal{F}_{\rho, \lambda}^{\sigma}\left(x-\ell_{1}\right)\right]^{2}}{\mathcal{F}_{\rho, \lambda}^{\sigma}\left(x-\ell_{1}\right)+\mathcal{F}_{\rho, \lambda}^{\sigma}\left(\ell_{2}-x\right)} \times \\
& \left(\int_{0}^{1} d_{q} \imath\right)^{1-\frac{1}{r}}\left(\left.\left.\int_{0}^{1}{ }^{r}{ }^{r}\right|_{\ell_{1}} \mathcal{D}_{q} f\left(\ell_{1}+\imath \mathcal{F}_{\rho, \lambda}^{\sigma}\left(x-\ell_{1}\right)\right)\right|^{r} d_{q} \imath\right)^{\frac{1}{r}} \\
& +\frac{q\left[\mathcal{F}_{\rho, \lambda}^{\sigma}\left(\ell_{2}-x\right)\right]^{2}}{\mathcal{F}_{\rho, \lambda}^{\sigma}\left(x-\ell_{1}\right)+\mathcal{F}_{\rho, \lambda}^{\sigma}\left(\ell_{2}-x\right)} \times \\
& \left(\int_{0}^{1} d_{q} \imath\right)^{1-\frac{1}{r}}\left(\left.\left.\int_{0}^{1} r^{r}\right|_{\ell_{1}} \mathcal{D}_{q} f\left(\ell_{2}+\imath \mathcal{F}_{\rho, \lambda}^{\sigma}\left(x-\ell_{2}\right)\right)\right|^{r} d_{q} \imath\right)^{\frac{1}{r}} \\
& \leq \frac{q\left[\mathcal{F}_{\rho, \lambda}^{\sigma}\left(x-\ell_{1}\right)\right]^{2}}{\mathcal{F}_{\rho, \lambda}^{\sigma}\left(x-\ell_{1}\right)+\mathcal{F}_{\rho, \lambda}^{\sigma}\left(\ell_{2}-x\right)} \times \\
& \left(\int_{0}^{1} d_{q} \imath\right)^{1-\frac{1}{r}}\left(\int_{0}^{1} \imath^{r}\left[\left.\left.(1-\imath)\right|_{\ell_{1}} \mathcal{D}_{q} f\left(\ell_{1}\right)\right|^{r}+\left.\left.\imath\right|_{\ell_{1}} \mathcal{D}_{q} f(x)\right|^{r}\right] d_{q}\right)^{\frac{1}{r}} \\
& +\frac{q\left[\mathcal{F}_{\rho, \lambda}^{\sigma}\left(\ell_{2}-x\right)\right]^{2}}{\mathcal{F}_{\rho, \lambda}^{\sigma}\left(x-\ell_{1}\right)+\mathcal{F}_{\rho, \lambda}^{\sigma}\left(\ell_{2}-x\right)} \times \\
& \left(\int_{0}^{1} d_{q} \imath\right)^{1-\frac{1}{r}}\left(\int_{0}^{1} \imath^{r}\left[\left.\left.(1-\imath)\right|_{\ell_{1}} \mathcal{D}_{q} f\left(\ell_{2}\right)\right|^{r}+\left.\left.\imath\right|_{\ell_{1}} \mathcal{D}_{q} f(x)\right|^{r}\right] d_{q} \imath\right)^{\frac{1}{r}}
\end{aligned}
$$




$$
\begin{aligned}
=\frac{q}{\mathcal{F}_{\rho, \lambda}^{\sigma}\left(x-\ell_{1}\right)+\mathcal{F}_{\rho, \lambda}^{\sigma}\left(\ell_{2}-x\right)}\left(\frac{1-q}{1-q^{r+2}}\right)^{1 / r} \times \\
\left\{\left[\mathcal{F}_{\rho, \lambda}^{\sigma}\left(x-\ell_{1}\right)\right]^{2} \sqrt[r]{\left.\left.\frac{q^{r}(1-q)}{1-q^{r+1}}\right|_{\ell_{1}} \mathcal{D}_{q} f\left(\ell_{1}\right)\right|^{r}+\left.\left.\right|_{\ell_{1}} \mathcal{D}_{q} f(x)\right|^{r}}\right. \\
\left.+\left[\mathcal{F}_{\rho, \lambda}^{\sigma}\left(\ell_{2}-x\right)\right]^{2} \sqrt[r]{\left.\left.\frac{q^{r}(1-q)}{1-q^{r+1}}\right|_{\ell_{1}} \mathcal{D}_{q} f\left(\ell_{2}\right)\right|^{r}+\left.\left.\right|_{\ell_{1}} \mathcal{D}_{q} f(x)\right|^{r}}\right\} .
\end{aligned}
$$

The proof is complete.

Corollary 5. Taking the limit when $q \rightarrow 1^{-}$in Theorem 5, we find

$$
\begin{aligned}
& \left|T_{f}\left(x, \ell_{1}, \ell_{2}\right)\right| \leq \frac{1}{(r+2)^{1 / r}\left(\mathcal{F}_{\rho, \lambda}^{\sigma}\left(x-\ell_{1}\right)+\mathcal{F}_{\rho, \lambda}^{\sigma}\left(\ell_{2}-x\right)\right)} \times \\
& \left\{\left[\mathcal{F}_{\rho, \lambda}^{\sigma}\left(x-\ell_{1}\right)\right] \sqrt[r]{\frac{1}{r+1}\left|f^{\prime}\left(\ell_{1}\right)\right|^{r}+\left|f^{\prime}(x)\right|^{r}}\right. \\
& \left.+\left[\mathcal{F}_{\rho, \lambda}^{\sigma}\left(\ell_{2}-x\right)\right]^{2} \sqrt[r]{\frac{1}{r+1}\left|f^{\prime}\left(\ell_{2}\right)\right|^{r}+\left|f^{\prime}(x)\right|^{r}}\right\} .
\end{aligned}
$$

Corollary 6. Additionally, if $\left.\right|_{\ell_{1}} \mathcal{D}_{q} f \mid \leq M$ for some $M>0$ in Theorem 5 , then we have

$$
\begin{aligned}
& \left|T_{f}\left(x, \ell_{1}, \ell_{2} ; q\right)\right| \\
& \leq M q \sqrt[r]{\left(\frac{q-1}{q^{r+2}-1}\right)\left(\frac{q^{r}(q-1)}{q^{r+1}-1}+1\right)}\left[\frac{\left[\mathcal{F}_{\rho, \lambda}^{\sigma}\left(x-\ell_{1}\right)\right]^{2}+\left[\mathcal{F}_{\rho, \lambda}^{\sigma}\left(\ell_{2}-x\right)\right]^{2}}{\mathcal{F}_{\rho, \lambda}^{\sigma}\left(x-\ell_{1}\right)+\mathcal{F}_{\rho, \lambda}^{\sigma}\left(\ell_{2}-x\right)}\right] .
\end{aligned}
$$

Remark 6. Letting $\rho=1, \lambda=0$ and $\sigma=(0,1,0,0, \cdots)$ in Raina's function, then we have $\mathcal{F}_{\rho, \lambda}^{\sigma}(y-x)=$ $y-x$ for all $x, y \in O$. In Corollary 6 , we find

$$
\left|T_{f}\left(x, \ell_{1}, \ell_{2} ; q\right)\right| \leq M q \sqrt[r]{\left(\frac{q-1}{q^{r+2}-1}\right)\left(\frac{q^{r}(q-1)}{q^{r+1}-1}+1\right)}\left[\frac{\left(x-\ell_{1}\right)^{2}+\left(\ell_{2}-x\right)^{2}}{\ell_{2}-\ell_{1}}\right] .
$$

\subsection{Quantum Estimates Using the Hypergeometric and Mittag-Leffler Functions}

As it was mentioned in the Preliminaries section, for a particular selection of choices for the parameters $\rho, \lambda$, and $\sigma$ in Raina's function (2), it is possible to find expressions using the hypergeometric function and the Mittag-Leffler function, between others, so we can establish similar results to those found in the Main Results section for these special functions.

\subsubsection{For the Hypergeometric Function}

Letting in (2) $\rho=1, \lambda=0$ and

$$
\sigma(k)=\frac{\left((\alpha)_{k}(\beta)_{k}\right)}{(\gamma)_{k}} \text { for } k=0,1,2, \ldots,
$$

then from Lemma 2, Theorem 3, Theorem 4, and Theorem 5, the following results hold. 
Lemma 3. Let $f: O \rightarrow \mathbb{R}$ be a $q$-differentiable function on $O^{\circ}$ with ${ }_{\ell_{1}} \mathcal{D}_{q} f$ considered continuous and integrable on $O$. Then the following identity holds:

$$
\begin{aligned}
& T_{f}\left(x, \ell_{1}, \ell_{2} ; q\right) \\
& \qquad \begin{array}{l}
\frac{q\left[F\left(\alpha, \beta ; \gamma ; x-\ell_{1}\right)\right]^{2}}{F\left(\alpha, \beta ; \gamma ; x-\ell_{1}\right)+F\left(\alpha, \beta ; \gamma ; \ell_{2}-x\right)} \int_{0}^{1} \imath_{\ell_{1}} \mathcal{D}_{q} f\left(\ell_{1}+\imath F\left(\alpha, \beta ; \gamma ; x-\ell_{1}\right) d_{q} \imath\right. \\
\quad+\frac{q\left[F\left(\alpha, \beta ; \gamma ; \ell_{2}-x\right)\right]^{2}}{F\left(\alpha, \beta ; \gamma ; \ell_{1}-x\right)+F\left(\alpha, \beta ; \gamma ; \ell_{2}-x\right)} \int_{0}^{1} \iota_{\ell_{1}} \mathcal{D}_{q} f\left(\ell_{2}+\imath F\left(\alpha, \beta ; \gamma ; x-\ell_{2}\right) d_{q} l,\right.
\end{array}
\end{aligned}
$$

where

$$
T_{f}\left(x, \ell_{1}, \ell_{2} ; q\right)=f(x)-\frac{1}{\mathcal{F}_{\rho, \lambda}^{\sigma}\left(x-\ell_{1}\right)+\mathcal{F}_{\rho, \lambda}^{\sigma}\left(\ell_{2}-x\right)} \int_{\ell_{1}}^{\ell_{1}+F\left(\alpha, \beta ; \gamma ; \ell_{2}-\ell_{1}\right)} f(\imath)_{\ell_{1}} d_{q} l
$$

Theorem 6. Let $f: O \rightarrow \mathbb{R}$ be a q-differentiable function on $O^{\circ}$ with ${ }_{\ell_{1}} \mathcal{D}_{q} f$ considered continuous and integrable on $O$. If $\left.||_{\ell_{1}} \mathcal{D}_{q} f\right|^{r}$ is generalized as $\phi$-convex on $O$ for $r>1$ and $\frac{1}{p}+\frac{1}{r}=1$, then the following inequality holds:

$$
\begin{gathered}
\left|T_{f}\left(x, \ell_{1}, \ell_{2} ; q\right)\right| \leq \frac{q}{\sqrt[p]{[p+1]}\left(F\left(\alpha, \beta ; \gamma ; x-\ell_{1}\right)+F\left(\alpha, \beta ; \gamma ; \ell_{2}-x\right)\right)} \times \\
\left\{\left[F\left(\alpha, \beta ; \gamma ; x-\ell_{1}\right)\right]^{2} \sqrt[r]{\frac{\left.\left.q\right|_{\ell_{1}} \mathcal{D}_{q} f\left(\ell_{1}\right)\right|^{r}+\left|\ell_{1} \mathcal{D}_{q} f(x)\right|^{r}}{1+q}}\right. \\
\left.+\left[F\left(\alpha, \beta ; \gamma ; \ell_{2}-x\right)\right]^{2} \sqrt[r]{\frac{\left.\left.q\right|_{\ell_{1}} \mathcal{D}_{q} f\left(\ell_{2}\right)\right|^{r}+\left|\ell_{\ell_{1}} \mathcal{D}_{q} f(x)\right|^{r}}{1+q}}\right\},
\end{gathered}
$$

where $[p+1]$ is the $q$-analogue of $p+1$.

Theorem 7. Let $f: O \rightarrow \mathbb{R}$ be a q-differentiable function on $O^{\circ}$ with ${ }_{\ell_{1}} \mathcal{D}_{q} f$ considered continuous and integrable on $O$. If $\left|\ell_{1} \mathcal{D}_{q} f\right|^{r}$ is generalized as $\phi$-convex on $O$, then for $r \geq 1$, the following inequality holds:

$$
\begin{aligned}
&\left|T_{f}\left(x, \ell_{1}, \ell_{2} ; q\right)\right| \leq \frac{q}{(1+q) \sqrt[r]{1+q+q^{2}}\left(F\left(\alpha, \beta ; \gamma ; x-\ell_{1}\right)+F\left(\alpha, \beta ; \gamma ; \ell_{2}-x\right)\right)} \times \\
&\left\{\left[F\left(\alpha, \beta ; \gamma ; x-\ell_{1}\right)\right]^{2} \sqrt[r]{q^{2}\left|\ell_{\ell_{1}} \mathcal{D}_{q} f\left(\ell_{1}\right)\right|^{r}+\left.\left.(1+q)\right|_{\ell_{1}} \mathcal{D}_{q} f(x)\right|^{r}}\right. \\
&\left.\left.+\left[F\left(\alpha, \beta ; \gamma ; \ell_{2}-x\right)\right)\right]^{2} \sqrt[r]{q^{2}\left|\ell_{1} \mathcal{D}_{q} f\left(\ell_{2}\right)\right|^{r}+\left.(1+q)||_{\ell_{1}} \mathcal{D}_{q} f(x)\right|^{r}}\right\} .
\end{aligned}
$$

Theorem 8. Let $f: O \rightarrow \mathbb{R}$ be a $q$-differentiable function on $O^{\circ}$ with ${ }_{\ell_{1}} \mathcal{D}_{q} f$ be continuous and integrable on O. If $\left.\left.\right|_{\ell_{1}} \mathcal{D}_{q} f\right|^{r}$ is generalized $\phi$-convex on $O$, then for $r \geq 1$, the following inequality holds:

$$
\begin{aligned}
&\left|T_{f}\left(x, \ell_{1}, \ell_{2} ; q\right)\right| \leq \frac{q}{F\left(\alpha, \beta ; \gamma ; x-\ell_{1}\right)+F\left(\alpha, \beta ; \gamma ; \ell_{2}-x\right)}\left(\frac{q-1}{q^{r+2}-1}\right)^{1 / r} \times \\
&\left\{\left[F\left(\alpha, \beta ; \gamma ; x-\ell_{1}\right)\right]^{2} \sqrt[r]{\left.\left.\frac{q^{r}(q-1)}{q^{r+1}-1}\right|_{\ell_{1}} \mathcal{D}_{q} f\left(\ell_{1}\right)\right|^{r}+\left.\left.(q+1)\right|_{\ell_{1}} \mathcal{D}_{q} f(x)\right|^{r}}\right. \\
&\left.+\left[F\left(\alpha, \beta ; \gamma ; \ell_{2}-x\right)\right]^{2} \sqrt[r]{\left.\left.\frac{q^{r}(q-1)}{q^{r+1}-1}\right|_{\ell_{1}} \mathcal{D}_{q} f\left(\ell_{2}\right)\right|^{r}+\left.\left.(q+1)\right|_{\ell_{1}} \mathcal{D}_{q} f(x)\right|^{r}}\right\} .
\end{aligned}
$$




\subsubsection{For the Mittag-Leffler Function}

Letting $\rho=\alpha, \operatorname{Re}(\alpha)>0, \lambda=1$ and $\sigma=(1,1,1, \cdots)$ in (2), then from Lemma 2, Theorem 3, Theorem 4 , and Theorem 5 , the following results hold.

Lemma 4. Let $f: O \rightarrow \mathbb{R}$ be a q-differentiable function on $O^{\circ}$ with ${ }_{\ell_{1}} \mathcal{D}_{q} f$ considered continuous and integrable on $O$. Then the following identity holds:

$$
\begin{aligned}
T_{f}\left(x, \ell_{1}, \ell_{2} ; q\right)=\frac{q\left[E_{\alpha}\left(x-\ell_{1}\right)\right]^{2}}{E_{\alpha}\left(x-\ell_{1}\right)+E_{\alpha}\left(\ell_{2}-x\right)} & \int_{0}^{1}{ }^{1} \ell_{1} \mathcal{D}_{q} f\left(\ell_{1}+\imath E_{\alpha}\left(x-\ell_{1}\right)\right) d_{q} \imath \\
& +\frac{q\left[E_{\alpha}\left(\ell_{2}-x\right)\right]^{2}}{E_{\alpha}\left(x-\ell_{1}\right)+E_{\alpha}\left(\ell_{2}-x\right)} \int_{0}^{1} \imath_{\ell_{1}} \mathcal{D}_{q} f\left(\ell_{2}+\imath E_{\alpha}\left(x-\ell_{2}\right) d_{q} \imath\right.
\end{aligned}
$$

where

$$
T_{f}\left(x, \ell_{1}, \ell_{2} ; q\right)=f(x)-\frac{1}{E_{\alpha}\left(x-\ell_{1}\right)+E_{\alpha}\left(\ell_{2}-x\right)} \int_{\ell_{1}}^{\ell_{1}+E_{\alpha}\left(\ell_{2}-\ell_{1}\right)} f(\imath)_{\ell_{1}} d_{q} l
$$

Theorem 9. Let $f: O \rightarrow \mathbb{R}$ be a q-differentiable function on $O^{\circ}$ with ${ }_{\ell_{1}} \mathcal{D}_{q} f$ considered continuous and integrable on $O$. If $\left|\ell_{1} \mathcal{D}_{q} f\right|^{r}$ is generalized as $\phi$-convex on $O$ for $r>1$ and $\frac{1}{p}+\frac{1}{r}=1$, then the following inequality holds:

$$
\begin{aligned}
&\left|T_{f}\left(x, \ell_{1}, \ell_{2} ; q\right)\right| \leq \frac{q}{\sqrt[p]{[p+1]}\left(E_{\alpha}\left(x-\ell_{1}\right)+E_{\alpha}\left(\ell_{2}-x\right)\right)} \times\left\{\left[E_{\alpha}\left(x-\ell_{1}\right)\right]^{2} \sqrt[r]{\frac{\left.\left.q\right|_{\ell_{1}} \mathcal{D}_{q} f\left(\ell_{1}\right)\right|^{r}+\left|\ell_{1} \mathcal{D}_{q} f(x)\right|^{r}}{1+q}}\right. \\
&\left.+\left[E_{\alpha}\left(\ell_{2}-x\right)\right]^{2} \sqrt[r]{\frac{\left.\left.q\right|_{\ell_{1}} \mathcal{D}_{q} f\left(\ell_{2}\right)\right|^{r}+\left.\left.\right|_{\ell_{1}} \mathcal{D}_{q} f(x)\right|^{r}}{1+q}}\right\},
\end{aligned}
$$

where $[p+1]$ is the $q$-analogue of $p+1$.

Theorem 10. Let $f: O \rightarrow \mathbb{R}$ be a q-differentiable function on $O^{\circ}$ with $\ell_{1} \mathcal{D}_{q} f$ considered continuous and integrable on $O$. If $\left.||_{1} \mathcal{D}_{q} f\right|^{r}$ is generalized as $\phi$-convex on $O$, then for $r \geq 1$, the following inequality holds:

$$
\begin{aligned}
\left|T_{f}\left(x, \ell_{1}, \ell_{2} ; q\right)\right| \leq & \frac{q}{(1+q) \sqrt[r]{1+q+q^{2}}\left(E_{\alpha}\left(x-\ell_{1}\right)+E_{\alpha}\left(\ell_{2}-x\right)\right)} \times \\
& \left\{\left[E_{\alpha}\left(x-\ell_{1}\right)\right] \sqrt[r]{q^{2}\left|\ell_{1} \mathcal{D}_{q} f\left(\ell_{1}\right)\right|^{r}+(1+q)\left|\ell_{1} \mathcal{D}_{q} f(x)\right|^{r}}\right. \\
& \left.\left.+\left[E_{\alpha}\left(\ell_{2}-x\right)\right)\right] \sqrt[r]{\left.\left.q^{2}\right|_{\ell_{1}} \mathcal{D}_{q} f\left(\ell_{2}\right)\right|^{r}+\left.\left.(1+q)\right|_{\ell_{1}} \mathcal{D}_{q} f(x)\right|^{r}}\right\} .
\end{aligned}
$$


Theorem 11. Let $f: O \rightarrow \mathbb{R}$ be a q-differentiable function on $O^{\circ}$ with ${ }_{\ell_{1}} \mathcal{D}_{q} f$ considered continuous and integrable on $O$. If $\left|\ell_{1} \mathcal{D}_{q} f\right|^{r}$ is generalized as $\phi$-convex on $O$, then for $r \geq 1$, the following inequality holds:

$$
\begin{aligned}
\left|T_{f}\left(x, \ell_{1}, \ell_{2} ; q\right)\right| \leq & \frac{q}{E_{\alpha}\left(x-\ell_{1}\right)+E_{\alpha}\left(\ell_{2}-x\right)}\left(\frac{q-1}{q^{r+2}-1}\right)^{1 / r} \times \\
& \left\{\left[E_{\alpha}\left(x-\ell_{1}\right)\right]^{2} \sqrt[r]{\left.\left.\frac{q^{r}(q-1)}{q^{r+1}-1}\right|_{\ell_{1}} \mathcal{D}_{q} f\left(\ell_{1}\right)\right|^{r}+\left.\left.(q+1)\right|_{\ell_{1}} \mathcal{D}_{q} f(x)\right|^{r}}\right. \\
& \left.+\left[E_{\alpha}\left(\ell_{2}-x\right)\right]^{2} \sqrt[r]{\left.\left.\frac{q^{r}(q-1)}{q^{r+1}-1}\right|_{\ell_{1}} \mathcal{D}_{q} f\left(\ell_{2}\right)\right|^{r}+\left.\left.(q+1)\right|_{\ell_{1}} \mathcal{D}_{q} f(x)\right|^{r}}\right\} .
\end{aligned}
$$

\section{Conclusions}

In the present study, certain new Ostrowski type inequalities have been established: Theorems 3-5, through the use of the identity established in Lemma 2, within the framework of quantum calculus and a class of parametrized special functions. From these results, particular cases for hypergeometric and Mittag-Leffler functions have been deduced. It is expected that from the results obtained, and following the methodology applied, additional special functions may also be evaluated. Future works can be developed in the area of numerical analysis and even contributions using quantum algorithms, using the theorems and corollaries presented. Finally, our results can be applied to derive some inequalities using special means. We omit their proof and the details are left to the interested reader.

Author Contributions: All authors contributed equally in the preparation of the present work: the theorems and corollaries M.J.V.-C., A.K., R.L. and J.E.H.H., the review of the articles and books cited M.J.V.-C., A.K., R.L. and J.E.H.H.; formal analysis M.J.V.-C., A.K., R.L. and J.E.H.H.; writing-original draft preparation and writing-review and editing M.J.V.-C., A.K., R.L. and J.E.H.H.

Funding: This research was funded by Dirección de Investigación from Pontificia Universidad Católica del Ecuador as a part of the research project entitled: Some inequalities using generalized convexity.

Acknowledgments: Miguel J. Vivas-Cortez thanks to Dirección de Investigación from Pontificia Universidad Católica del Ecuador and Jorge E. Hernández Hernández thanks to Consejo de Desarrollo Científico, Humanístico y Tecnológico from Universidad Centroccidental Lisandro Alvarado (Venezuela) for the technical support given in the development of the present article, the first under the project entitled: Algunas desigualdades de funciones convexas generalizadas (Some inequalities of generalized convex functions). Also, all the authors thanks to the appointed referees for their appropriate comments in the evaluation of this work and to the editorial team from Symmetry for the serious and responsible work performed.

Conflicts of Interest: The authors declare no conflict of interest.

\section{References}

1. Jackson, F.H. On a q-definite integrals. Q. J. Pure Appl. Math. 1910, 41, 193-203.

2. Ernst, T. The different tongues of q-calculus. Proc. Eston. Acad. Sci. 2008, 57, 81-99. [CrossRef]

3. Ernst, T. A Comprehensive Treatment of q-Calculus; Springer: Basel, Switzerland, 2012.

4. Gauchman, H. Integral Inequalities in q-Calculus. Comp. Math. Appl. 2004, 47, 281-300. [CrossRef]

5. Kac, V.; Cheung, P. Quantum Calculus; Universitext Springer: New York, NY, USA, 2002.

6. Ismail, M.E.H. Classical and Quantum Orthogonal Polynomials in One Variable; Cambridge University Press: Cambridge, UK, 2005.

7. Ismail, M.E.H.; Mansour, Z.C.I. q-analogues of Freud weights and nonlinear difference equations. Adv. Appl. Math. 2010, 45, 518-547. [CrossRef]

8. Brahim, K.; Taf, S.; Nefzi, B. New Integral Inequalities in Quantum Calculus. Int. J. Anal. Appl. 2015, 7, 50-58.

9. Mirković, T.Z.; Tričković, S.B.; Stanković, M.S. Opial inequality in q-calculus. J. Ineq. Appl. 2018, $2018,1-8$. [CrossRef]

10. Niculescu, C.P. An invitation to convex function theory. In Order Structures in Functional Analysis; Editura Academiei Romane: Bucharest, Romania, 2001.

11. Bennett, C.; Sharpley, R. Interpolation of Operators; Academic Press: Boston, MA, USA, 1998. 
12. Nguyen, N.A.; Gulan, M.; Olaru, S.; Averbe, P.R. Convex Lifting: Theory and Control Applications. IEEE Trans. Autom. Control 2010, 63, 1-16. [CrossRef]

13. Mititelu, Ş.; Trenţă, S. Efficiency conditions in vector control problems governed by multiple integrals. J. Appl. Math. Comp. 2018, 57, 647-665. [CrossRef]

14. Trenţă, S. On a New Class of Vector Variational Control Problems. Num. Funct. Anal. Optimi. 2018, 39, 1594-1603. [CrossRef]

15. Trenţă, S. KT-geodesic pseudoinvex control problems governed by multiple integrals. J. Nonlin. Conv. Anal. 2019, 20, 73-84.

16. Jensen, J.L.W.V. Om konvexe Funktioner og Uligheder mellen Middelvaerdier. Nyt. Tidsskr. Math. $1905,16,49-69$.

17. Jensen, J.L.W.V. Sur les fonctions convexes et les inegalités entre les valeurs moyennes. Acta Math. 1906, 30, 175-193. [CrossRef]

18. Alomari, M.; Darus, M.; Dragomir, S.S.; Cerone, P. Ostrowski type inequalities for functions whose derivatives are s-convex in the second sense. Appl. Math. Lett. 2010, 23, 1071-1076. [CrossRef]

19. Akdemir, A.O.; Özdemir, M.E. Some Hadamard-type inequalities for coordinated P-convex functions and Godunova-Levin functions. AIP Conf. Proc. 2010, 1309, 7-15.

20. Liu, W.; Wen, W.; Park, J. Hermite-Hadamard type inequalities for MT-convex functions via classical integrals and fractional integrals. J. Nonlinear Sci. Appl. 2016, 9, 766-777. [CrossRef]

21. Hernández Hernández, J.E. On Some New Integral Inequalities Related with the Hermite-Hadamard Inequality via h-Convex Functions. MAYFEB J. Math. 2017, 4, 1-12

22. Hernández Hernández, J.E. On $\log -\left(m, h_{1}, h_{2}\right)$-convex functions and related integral inequalities. Int. J. Open Prob. Compt. Math. 2019, 12, 43-59.

23. Vivas-Cortez, M.J.; García, C.; Hernández Hernández, J.E. Ostrowski-Type Inequalities for Functions Whose Derivative Modulus is Relatively $\left(m, h_{1}, h_{2}\right)$-Convex. Appl. Math. Inf. Sci. 2019, 13, 369-378.

24. Vivas-Cortez, M.J.; García, C.; Hernández Hernández, J.E. Ostrowski Type Inequalities for Functions Whose Derivative Modulus is Relatively Convex. Appl. Math. Inf. Sci. 2019, 13, 1-7.

25. Vivas-Cortez, M.J.; Hernández Hernández, J.E. Hermite-Hadamard inequalities type for Raina's fractional integral operator using $\eta$-convex functions. Revista Matemática: Teoría y Aplicaciones 2019, 26, 1-19.

26. Vivas-Cortez, M.J.; Hernández Hernández, J.E. Ostrowski and Jensen type inequalities via $(s, m)$-convex functions in the second sense. Boletin de la Sociedad Matemática Mexicana 2019, in press.

27. Dragomir, S.S.; Rassias, T.M. Ostrowski Type Inequalities and Applications in Numerical Integration; Springer: Cham, The Netherlandz, 2002.

28. Alomari, M.; Darus, M. Some Ostrowski type inequalities for quasi-convex functions with applications to special means. RGMIA Res. Rep. Coll. 2010, 13, 1-15.

29. Cerone, P.; Dragomir, S.S. Ostrowski type inequalities for functions whose derivative satisfy certain convexity assumptions. Demonstr. Math. 2004, 37, 299-308. [CrossRef]

30. Hernández Hernández, J.E. Ostrowski type fractional integral operator inequalities for $\left(m, h_{1}, h_{2}\right)$-convex functions. MAYFEB J. Math. 2017, 4, 13-28.

31. Noor, M.A.; Awan, M.U.; Noor, K.I. Quantum Ostrowski inequalities for $q$-differentiable convex functions. J. Math. Inequal. 2016, 10, 1013-1018. [CrossRef]

32. Tomar, M.; Agarwal, P.; Jleli, M.; Samet, B. Certain Ostrowski type inequalities for generalized s-convex functions. J. Nonlinear Sci. Appl. 2017, 10, 5947-5957. [CrossRef]

33. Noor, M.A. Some new classes of non-convex functions. Nonlinear Funct. Anal. Appl. 2006, 11, $165-171$.

34. Raina, R.K. On generalized Wright's hypergeometric functions and fractional calculus operators. East Asian Math. J. 2005, 21, 191-203.

35. Vivas-Cortez, M.J.; Liko, R.; Kashuri, A.; Hernández Hernández, J.E. New quantum estimates of trapezium-type inequalities for generalized $\phi$-convex functions. Mathematics 2019, in press. [CrossRef]

36. Alp, N.; Sarikaya, M.Z.; Kunt, M.; İscan, İ. q-Hermite Hadamard inequalities and quantum estimates for midpoint type inequalities via convex and quasi-convex functions. J. King Saud Univ. Sci. 2018, 30, 193-203. [CrossRef]

37. Liu, W.J.; Zhuang, H.F. Some quantum estimates of Hermite-Hadamard inequalities for convex functions. J. Appl. Anal. Comput. 2017, 7, 501-522. 
38. Noor, M.A.; Noor, K.I.; Awan, M.U. Some quantum estimates for Hermite-Hadamard inequalities. Appl. Math. Comput. 2015, 251, 675-679. [CrossRef]

39. Tariboon, J.; Ntouyas, S.K. Quantum integral inequalities on finite intervals. J. Inequal. Appl. 2014, 2014, 1-13. [CrossRef]

40. Zhuang, H.; Liu, W.; Park, J. Some quantum estimates of Hermite-Hadamard inequalities for quasi-convex functions. Mathematics 2019, 7, 152. [CrossRef]

(C) 2019 by the authors. Licensee MDPI, Basel, Switzerland. This article is an open access article distributed under the terms and conditions of the Creative Commons Attribution (CC BY) license (http://creativecommons.org/licenses/by/4.0/). 